\title{
Análise qualitativa do Plano Municipal de Saúde de São Paulo, Brasil - Com o apoio do software webQDA ${ }^{\circledR}$
}

\author{
Alexandra Bulgarelli do Nascimento' \\ ${ }^{1}$ Centro Universitário Senac - SP, Brasil | abnascimento@usp.br \\ http://orcid.org/0000-0003-4081-8782
}

\begin{abstract}
Resumo: Introdução: O Sistema Único de Saúde, no Brasil, tem o objetivo de assistir à população em suas necessidades em saúde. Para tanto, os municípios contam com o Plano Municipal de Saúde (PMS) para planejar e monitorar as ações implementadas. Objetivos: Analisar como o PMS de São Paulo reconhece as necessidades em saúde da população, em especial, de grupos sociais vulneráveis, e analisar o apoio do software webQDA ${ }^{\circledR}$ na organização e análise do grande volume de dados qualitativos provenientes do PMS de São Paulo. Método: Estudo qualitativo com análise de conteúdo de Bardin e apoio do webQDA ${ }^{\circledR}$, para desvelar o significado do PMS de São Paulo de 2014-2017. Resultados e Discussão: O PMS em arquivo word foi inserido no webQDA ${ }^{\circledR}$ como fonte interna. A árvore de codificação das categorias empíricas foi criada, com a inclusão dos códigos: 'Vulnerabilidades Sociais' e 'Resposta às Necessidades em Saúde', e a análise de conteúdo de Bardin, foi realizada evidenciando que o PMS de 2014-2017 está centrado em ideias de natureza funcionalistas e pautado na produtividade dos serviços, em detrimento da identificação das heterogêneas necessidades em saúde, em especial, de grupos sociais vulneráveis. Conclusões: O PMS de 2014-2017 trouxe alguns objetivos relacionados à presença de vulnerabilidades na cidade de São Paulo, o que é um avanço, apesar de quantitativamente possuir reduzida representatividade frente à maioria dos objetivos propostos e direcionados para responder à ocorrência de doenças e agravos, desarticulados dos perfis epidemiológicos dos diferentes grupos sociais. O webQDA ${ }^{\circledR}$ apoiou o desenvolvimento deste estudo, ao permitir o manejo de grande volume de dados qualitativos, bem como o remanejamento de trechos do PMS entre os códigos, como proposto pela análise de conteúdo de Bardin que estabelece a necessidade de exaustão da análise dos fragmentos extraídos para composição das unidades de significado e explicação do fenômeno sob análise.
\end{abstract}

Palavras-chave: Pesquisa Qualitativa; Planejamento em Saúde; Sistema Único de Saúde; Avaliação das Necessidades de Cuidados de Saúde; Vulnerabilidade Social.

Qualitative Analysis of the Municipal Health Plan of Sao Paulo, Brazil - With the Support of the webQDA® Software

Abstract: Introduction: The Unified Health System, in Brazil, aims to assist the population in their health needs. To this end, the municipalities rely on the Municipal Health Plan (PMS) to plan and monitor the actions implemented. Objectives: To analyze how the São Paulo PMS recognizes the health needs of the population, in particular, vulnerable social groups, and to analyze the support of the webQDA® software in the organization and analysis of the large volume of qualitative data from the São Paulo PMS. Method: Qualitative study with content analysis by Bardin and support from webQDA®, to unveil the meaning of the São Paulo PMS from 2014-2017. Results and Discussion: The PMS in word file was inserted in webQDA® as an internal source. The coding tree for the empirical categories was created, with the inclusion of the codes: 'Social Vulnerabilities' and 'Response to Health Needs', and Bardin's content analysis was carried out showing that the 2014-2017 PMS is centered on ideas of a functionalist nature and guided by the productivity of services, to the detriment of the identification of heterogeneous health needs, in particular, of vulnerable social groups. Conclusions: The 2014-2017 PMS brought some objectives related to the presence of vulnerabilities in the city of São Paulo, which is an improvement, despite having a reduced representation in relation to most of the objectives proposed and directed to respond to the occurrence of diseases and injuries, disjointed from the epidemiological profiles of different social groups. WebQDA® supported the development of this study, by allowing the handling of a large volume of qualitative data, as well as the relocation of parts of the PMS between codes, as proposed by Bardin's content analysis, which establishes the need to exhaust the analysis of fragments extracted to compose the units of meaning and explanation of the phenomenon under analysis.

Keywords: Qualitative Research; Health Planning; Unified Health System; Needs Assessment; Social Vulnerability. 


\section{Introdução}

O Sistema Único de Saúde (SUS) tem o objetivo de prover, universalmente, ações de promoção, prevenção, restauração, reabilitação e cuidados paliativos (Brasil, 2009). Para tanto, espera-se que o SUS garanta acesso, equidade, qualidade, efetividade e sustentabilidade do Sistema (Ducan et al., 2015).

O SUS, como política de Estado, ampliou as práticas assistenciais e gerenciais ao conceber a saúde como interligada à qualidade de vida e bem-estar das pessoas, o que significa a necessidade de considerar as condições de habitação, transporte, saneamento e trabalho, além de outros aspectos que determinam os diferentes perfis do modo de viver, adoecer e morrer de distintos grupos sociais (Duncan et al., 2015).

Os gestores locais, no SUS, devem organizar a assistência à saúde, o que - muitas vezes - ocorre por meio da ação isolada da oferta de serviços de saúde de forma desarticulada da identificação das necessidades em saúde - o que denota descompasso entre a demanda e a oferta, acentuando as desigualdades alocativas de recursos e deficiência no atendimento às necessidades em saúde (Nascimento, 2015; Santos \& Giovanella, 2014), em especial, de grupos sociais vulneráveis.

O Plano Municipal de Saúde (PMS) é um instrumento de gestão local, cujo objetivo é monitorar e planejar as ações, com vistas a atenderem às necessidades em saúde da população, garantindo que ações articuladas sejam providas adequadamente (Nascimento \& Egry, 2017).

O PMS tem vigência de quatro anos e busca mapear a situação de saúde da população, bem como estabelecer prioridades, objetivos e metas, visando contribuir para a efetiva atenção à saúde (Brasil, 2009), o que exige que considere as necessidades em saúde de grupos sociais vulneráveis (Nascimento \& Egry, 2017).

Para atender ao seu objetivo, o PMS deve reconhecer a existência de heterogêneas necessidades em saúde, que potencializam a sua utilização ao organizar o planejamento em saúde local centrado na efetividade da assistência prestada e, consequentemente, a qualidade da atenção percebida pela população (Albuquerque et al., 2011).

O PMS se coloca como potente para contribuir para minimizar as iniquidades em saúde, por meio do mapeamento das heterogêneas necessidades em saúde, bem como da sistematização de ações, objetivos e metas, que visam agregar efetividade à atenção à saúde, em especial, dos grupos sociais vulneráveis (Bueno et al., 2013).

Para que essa perspectiva de utilização do PMS se confirme no cotidiano das secretarias de saúde, os gestores devem pautar as suas práticas no modelo explicativo do processo saúde-doença centrado na determinação social - o que implica a mudança de paradigma em relação à compreensão dos fenômenos relacionados ao modo de adoecer e morrer das pessoas (Breilh, 2019).

Essa mudança de paradigma não está presente na realidade das práticas de gestão em saúde pública local, como concluiu um estudo realizado em quatro municípios brasileiros que demonstrou o distanciamento entre as práticas de gestão local, e a determinação social do processo saúde-doença, denotando limitação para a resposta efetiva do sistema de saúde sobre as necessidades da população, em especial de grupos sociais vulneráveis (Nascimento \& Egry, 2017).

Diante da complexidade do tema proposto, optou-se pelo uso do software Web Qualitative Data Analysis (webQDA ${ }^{\circledR}$ ) para apoiar a análise dos dados qualitativos (Minayo \& Costa, 2019), visto o grande volume e densidade teórica presente neles.

O software webQDA ${ }^{\circledR}$ possibiltou a importação do arquivo referente ao PMS na íntegra, bem como a proposição de categorias empíricas para análise de conteúdo de Bardin (Bardin, 2015), por meio de códigos inseridos no próprio software para organização dos dados coletados. 
As perguntas de pesquisa deste estudo foram: a) Como o PMS de São Paulo reconhece as necessidades em saúde da população, em especial de grupos sociais vulneráveis? e b) O software webQDA ${ }^{\circledR}$ apoiou a organização e análise do grande volume de dados documentais?

Portanto, os objetivos deste estudo foram analisar como o PMS de São Paulo reconhece as necessidades em saúde da população, em especial, de grupos sociais vulneráveis, bem como analisar o apoio do software webQDA ${ }^{\circledR}$ na organização e análise do grande volumee de dados qualitativos provenientes do PMS de São Paulo.

\section{Metodologia}

Estudo documental, exploratório-descritivo, retrospectivo e com abordagem qualitativa, realizado em junho de 2020, e fundamentado na Teoria da Intervenção Práxica da Enfermagem em Saúde Coletiva (TIPESC), a partir do referencial teórico do MaterialismoHistórico e Dialético (Egry, 1996).

A TIPESC é sistematizada, a partir da análise crítica da realidade, em cinco etapas: 1) captação da realidade objetiva, 2) interpretação da realidade captada, 3) projeto de intervenção sobre a realidade, 4) intervenção propriamente dita, e 5) reinterpretação da realidade (Egry, 1996), sendo que neste estudo foram utilizadas as duas primeiras etapas propostas.

O cenário foi o município de São Paulo, que se caracteriza como a maior cidade e economia brasileira.

Os PMS referentes aos quadriênios de 2014-2017 e 2018-2021 foram analisados após localizados na plataforma web de domínio público do Ministério da Saúde: Sistema de Apoio à Elaboração do Relatório de Gestão (SARG-SUS).

Ambos PMS, convertidos para arquivo word, foram inseridos no software webQDA® como fonte interna, totalizando mais de 600 páginas para análise documental.

O processo de análise dos dados ocorreu com a colaboração de quatro pesquisadores que constituíram uma equipe de trabalho e pesquisa (ETP).

Os dados extraídos foram organizados no software webQDA®, por meio das categorias empíricas: 'Vulnerabilidades Sociais' e 'Resposta às Necessidades em Saúde' - as quais foram propostas em consenso pela ETP, a partir do conhecimento sobre objetivo do PMS como instrumento de gestão local, bem como por possuírem adesão ao problema de pesquisa presente no primeiro objetivo deste estudo: "Analisar como o PMS de São Paulo reconhece as necessidades em saúde da população, em especial, de grupos sociais vulneráveis.".

O software webQDA® teve um papel de grande valia, ao permitir a inserção de um vasto material textual, bem como a organização e inserção dos trechos selecionados nas categorias empíricas propostas - o que possibilitou a decisão da ETP sobre a análise do PMS do quadriênio de 2014-2017, uma vez que o PMS de 2018-2021 não dispôs de conteúdo relevante alinhado ao problema de pesquisa proposto.

A extração dos dados dos PMS foi realizada, por três dos quatro pesquisadores da ETP, os quais realizaram a análise de conteúdo preconizada por Bardin, que ao se utilizar da hermenêutica crítica, estrutura a elaboração e análise das unidades de significado, visando evidenciar a essência do fenômeno de interesse (Bardin, 2015).

Esse processo exigiu dos pesquisadores a reanálise pormenorizada dos dados em diferentes momentos, inclusive quando do consenso sobre a consolidação dos conteúdos nas respectivas categorias empíricas, bem como da atuação do quarto pesquisador da ETP na fase final de revisão de todos os conteúdos extraídos e organizados nas categorias, o que foi facilitado com o uso do software webQDA®. 
Esse estudo ao propor o manejo de dados secundários e de domínio público, dispensou a sua apreciação por um Comitê de Ética em Pesquisa - como ampara a Resolução do Conselho Nacional de Saúde no 510/2016 (Brasil, 2016).

\section{Resultados e Discussão}

Para operacionalização do SUS com qualidade é imprescindível o reconhecimento e avaliação das necessidades em saúde, e das vulnerabilidades dos grupos sociais dos territórios geopolíticos (Egry, 2018).

A análise do PMS de São Paulo referente ao quadriênio 2014-2017 permitiu identificar, a presença de 1149 objetivos específicos que nortearam o planejamento das ações propostas com suas respectivas metas e indicadores.

Desse total de objetivos específicos, 1,7\% (20) deles versaram sobre mecanismos de enfrentamento de vulnerabilidades, cujas evidências foram organizadas na categoria empírica 'Vulnerabilidades Sociais' e estão descritas a seguir:

Implementar Programa Consultório na Rua (CnaR), integrando as ações de suas equipes com as de ESF, UBSI, CAPS adulto, CAPS infantil e CAPS álcool e drogas, a fim de incluir no SUS pessoas em situação de rua e alta vulnerabilidade. [grifo nosso].

Reduzir a vulnerabilidade da juventude negra e moradora da periferia, criando estratégias de ampliação de direitos, enfrentamento ao racismo e prevenção da violência nos territórios atingidos pelos altos índices de mortalidade. [grifo nosso].

Monitorar a qualidade da água para consumo humano e estabelecer novas tecnologias de abordagem em conjunto com as CRS (SUVIS/UBS) e território, levando em consideração critérios epidemiológicos de risco e vulnerabilidade. [grifo nosso].

Implementar o controle de vetores e reservatórios responsáveis pela transmissão das zoonoses de importância em saúde pública no município de São Paulo, por meio da reestruturação do processo de trabalho das equipes de vigilância em saúde ambiental das Supervisões de Vigilância em Saúde, de forma a desenvolver ações de base territorial que considerem os critérios de risco, vulnerabilidades, potencialidades e ações locais articuladas. [grifo nosso].

Intervir nos fatores determinantes e condicionantes das doenças crônicas não-transmissíveis, de acordo com o perfil de vulnerabilidade e com base em critérios epidemiológicos. [grifo nosso].

Realizar ações em conjunto com a educação por meio do Programa Saúde na Escola e em áreas de grande vulnerabilidade por ações da Estratégia Saúde da Família, Abrigos de Menores e Associações de Comunidades de Imigrantes. [grifo nosso].

Implementar a atenção à saúde e o cuidado à população em situação de rua e vulnerabilidade. [grifo nosso].

Promover grupos semanais com usuários nas Unidades da região do Itaim Paulista, tendo em vista a vulnerabilidade às doenças, enfermidades graves e crônicas. [grifo nosso].

Favorecer o acesso, acolhimento, classificação de risco e vulnerabilidade à gestante, nas ações da Unidade Básica de Saúde. [grifo nosso].

Classificar e avaliar a vulnerabilidade da família deste recém-nascido e ampliar e promover a integração destas ações com as Maternidades da região que atendem este recém-nascido. [grifo nosso]. 
Criar Núcleos de Vigilância em Saúde nas Unidades, visando: intensificar a busca ativa de hanseníase e tuberculose intra e extra muros, agregando equipe da Unidade Básica de Saúde, Estratégia Saúde da Família e equipamentos da educação; fortalecer treinamento e ações de busca ativa em locais de maior vulnerabilidade; e capacitar os profissionais para identificar e tratar adequadamente a sífilis. [grifo nosso].

Implantar novos serviços e Unidades na região, em área de alta vulnerabilidade social, com ampliação da rede de atenção ampliando o acesso à assistência à saúde, garantindo a integralidade do cuidado. [grifo nosso].

Aumentar e assegurar a rede de apoio ao usuário em situação de risco e vulnerabilidade social. [grifo nosso].

Realizar cuidados complexos para crianças e adolescentes com psicopatologias graves e com riscos psicopatológicos simultâneos a vulnerabilidade socioeconômica e desestruturação familiar em território descoberto. [grifo nosso].

Realizar capacitações específicas sobre identificação de vulnerabilidades e vinculação da gestante às Unidade Básicas de Saúde. [grifo nosso].

Favorecer cuidados com crianças e adolescentes com graves psicopatologias, em crises, ou riscos às mesmas, por alta vulnerabilidade socioeconômica e desestruturação familiar. [grifo nosso].

Promover a esterilização cirúrgica de cães e gatos e identificação do responsável, por meio de serviços tercerizados e equipes próprias, especialmente nas regiões com maior índice de exclusão social. [grifo nosso].

Propiciar cuidados aos usuários de crack e outras drogas com alta vulnerabilidade em ambiente terapêutico protegido. [grifo nosso].

O PMS analisado apresentou objetivos relacionados ao enfrentamento de vulnerabilidades e identificação de grupos sociais com necessidades específicas, no entanto, eles se mostraram inexpressivos frente ao número total de objetivos propostos e que, em sua maioria, estiveram centrados na resposta à ocorrência de doenças e agravos, processos de trabalho e produção do cuidado em saúde - cujas evidências foram organizadas na categoria empírica "Resposta às Necessidades em Saúde" e estão apresentadas a seguir:

Estruturar a atenção à saúde às pessoas com hanseníase, tuberculose $\mathbf{e}$ hepatites virais $\mathrm{B}$ e $\mathrm{C}$.

Aprimorar a gestão da fila de espera da Atenção Especializada Ambulatorial.

Qualificar 0 atendimento de urgência e emergência, adotando nova modalidade de assistência - Unidades de Pronto Atendimento.

Avaliar em tempo oportuno a quantidade de médicos necessários, contratados e presentes na rede municipal de saúde.

Esses objetivos identificados no PMS para responder às necessidades em saúde da população são importantes para o planejamento em saúde. Entretanto, apresentam limites na sua efetividade, ao não estarem atrelados à realidade dos diferentes territórios que compõem o município e que abarcam grupos sociais específicos, com necessidades em saúde heterogêneas.

É fundamental pensar para além da oferta de ações e serviços, uma vez que o atendimento às necessidades em saúde exige compreensão aprofundada e pormenorizada da realidade das diferentes coletividades que compõem o território (OPAS, 2011; Nascimento, 2015). 
A construção desses resultados foi facilitada com o uso do software webQDA ${ }^{\circledR}$, que ao propor a organização dos dados qualitativos analisados em códigos, possibilitou que fossem reanalisados com maior facilidade, contribuindo para otimizar o tempo de execução do estudo.

Esse processo reiterou o PMS como uma ferramenta gerencial relevante para reorientar a gestão em saúde local, sendo primordial que esteja lastreada em bases teóricoconceituais que possibilitem a articulação dos perfis de saúde e doença aos de reprodução social, ou seja, que esteja ancorada na teoria interpretativa da determinação social do processo saúde-doença, identificando os grupos sociais, vulnerabilidades e necessidades diferenciadas, contribuindo para a adoção de práticas de gestão em saúde comprometidas com a equidade e integralidade do cuidado (Nascimento \& Egry, 2017).

A readequação dos instrumentos de gestão local, como do PMS, além da qualificação dos trabalhadores envolvidos no processo de captação das necessidades em saúde dos grupos sociais heterogêneos, bem como dos tomadores de decisão - representados pelos assessores das secretarias de saúde municipal e pelo próprio Secretário Municipal de Saúde, são essenciais.

Isso porque, esses instrumentos de gestão, como o PMS, estão alicerçados sobre as diretrizes da Epidemiologia Clássica e do modelo Multicausal de compreensão do processo saúde-doença - limitando o atendimento às necessidades em saúde, o que foi evidenciado neste estudo, por meio da ausência de propostas para identificar e localizar no território os grupos sociais vulneráveis presentes no município de São Paulo, os quais têm necessidades em saúde específicas e precisam ser respondidas de forma diferenciada pelo SUS.

Isso foi reiterado no PMS analisado, à medida que a categoria empírica 'Resposta às Necessidades em Saúde' denotou a presença de indicadores de resultados atrelados à implementação de ações de natureza funcionalista, ou seja, centradas na oferta da capacitação de equipes e serviços de saúde, como significado de resposta ao objetivo específico proposto no PMS, o que está evidenciado nos trechos a seguir:

Objetivo Específico: Reduzir a vulnerabilidade da juventude negra e moradora da periferia, criando estratégias de ampliação de direitos, enfrentamento ao racismo e prevenção da violência nos territórios atingidos pelos altos índices de mortalidade. [grifo nosso].

Indicador(es) de Resultado: 1- Percentual de jovens bolsitas capacitados entre os previstos; 2- Percentual de profissionais capacitados entre os previstos. [grifo nosso].

Objetivo Específico: Implementar a atenção à saúde e o cuidado à população em situação de rua e vulnerabilidade. [grifo nosso].

Indicador(es) de Resultado: Proporção entre o $\mathrm{n}^{\circ}$ de usuários em situação de rua atendidos no período em relação ao período anterior. [grifo nosso].

Objetivo Específico: Realizar capacitações específicas sobre identificação de vulnerabilidades e vinculação da gestante às Unidade Básicas de Saúde. [grifo nosso].

Indicador(es) de Resultado: 1- № de profissionais capacitados/previstos $\mathrm{x}$ 100; e 2 - Proporção de gestantes identificadas para vinculação à UBS.

Objetivo Específico: Propiciar cuidados aos usuários de crack e outras drogas com alta vulnerabilidade em ambiente terapêutico protegido. [grifo nosso].

Indicador(es) de Resultado: CAPS III Infantil implantado [...]. [grifo nosso].

A análise dos indicadores de resultados propostos para os objetivos específicos do PMS de 2014-2017, denota a urgência da adoção da concepção de saúde socialmente determinada como caminho para responder de forma efetiva às necessidades em saúde da população. 
A análise qualitativa dos objetivos específicos junto com os indicadores foi oportunizada por uma das funcionalidades do software webQDA®, e escancara a natureza funcionalista do monitoramento das ações propostas no PMS, as quais estiveram centradas na concepção do processo de saúde pautada na doença, procedimentos, implantação de serviços e capacitação de equipes - as quais apesar de importantes, são insuficientes para garantir a resposta às diferentes necessidades em saúde que emergem de realidades desiguais.

Para garantir a efetividade dos objetivos específicos propostos no PMS, os indicadores de resultados, ao invés de descreverem a realidade, por meio do número de profissionais capacitados, serviços implantados, procedimentos realizados, entre outros - devem possuir demonstrar o quanto as ações propostas impactaram e geraram transformação da realidade, isso porque a compreensão sobre as formas de adoecimento e morte da população transcendem a doença e são socialmente determinadas (Gatto-Júnior et al., 2015).

Para atuar no SUS não são suficientes conhecimentos e habilidades relacionadas ao ser humano biológico e à clínica como maneira de intervir. Nessa prática profissional é preciso compreender o nexo bio-psíquico-social; o processo saúde-doença socialmente determinado; e a Epidemiologia Crítica, os quais estão alicerçados sobre os pressupostos da Saúde Coletiva (Egry, 2018).

A gestão local comprometida com o atendimento efetivo às necessidades em saúde, captação e reconhecimento das diferentes realidades presentes no território (Hino et al., 2009) subsidiam as práticas gerenciais efetivas em resposta às heterogêneas necessidades em saúde e, em especial, de grupos sociais vulneráveis.

A mudança na compreensão de como ocorre o processo de saúde e de doença, implica o aumento significativo do trabalho e do desenvolvimento de competências dos profissionais, tanto assistenciais, como gerenciais (Martins, Egry \& Apostólico, 2015), uma vez que eles serão exigidos a atuarem de forma analítica em seus respectivos escopos de trabalho, denotando a necessidade de investimento maciço em qualificação professional, o que não foi evidenciado no PMS analisado.

No território, os fenômenos sociais expressos nos perfis de saúde se manifestam de forma explícita, demandando desses profissionais competências para o reconhecimento das necessidades em saúde e para o enfrentamento das vulnerabilidades (Martins, Egry \& Apostólico, 2015), reforçando a necessidade de mudanças nos instrumentos de gestão, bem como na qualificação dos trabalhadores e tomadores de decisão (Nascimento \& Egry, 2017).

Este trabalho avançou ao vocalizar a urgência da mudança de paradigma do modelo explicativo do processo do adoecer e morrer nas práticas de gestão em saúde local, uma vez que ele não se dá linearmente, mas de forma heterogênea a depender, principalmente, da posição social ocupada pelas pessoas.

Além disso, ele demonstra que apesar de discreta, houve a preocupação da gestão local no quadriênio 2014-2017, em se aproximar de conceitos que traduzem maior densidade à compreensão do processo saúde-doença, como ao propor ações pautadas na vulnerabilidade social, o que infelizmente não teve continuidade no PMS de 2018-2021, demonstrando retrocesso na política municipal de saúde.

A relevância de uma formação diferenciada dos profissionais - sejam eles assistenciais ou gerenciais - de forma a compreenderem o processo de saúde e de doença como socialmente determinado, visando subsidiar condições que possibilitem a ruptura de realidades limitantes, conjuntamente, à adoção de ações de governança local, que utilizem iniciativas intersetoriais compreendidas de forma integrada e articulada é fundamental para o atendimento às necessidades em saúde das pessoas. (Larsen et al., 2014; Santos \&; Giovanella, 2014). 


\section{Conclusões}

O software webQDA ${ }^{\circledR}$ foi essencial no desenvolvimento deste estudo à medida que possibilitou o manejo de grande volume de dados qualitativos, que exigiram da ETP a reanálise contínua dos códigos inseridos e dos trechos a eles atribuídos, otimizando o tempo dispendido para a execução da pesquisa.

Ademais, o software permitiu embasar a seleção de apenas um dos PMS propostos para constituir o escopo de análise do estudo, frente ao esvaziamento de conteúdo apresentado no PMS de 2018-2021, denotando maior rigor metodológico na sua execução, o que por outro lado também se constituiu como uma limitação deste estudo que num primeiro momento buscava comparar os conteúdos dos PMS de dois quadriênios, o que não foi possível e demonstra uma lacuna para o desenvolvimento de estudos futuros.

Outro ganho foi diante das evidências provenientes da análise do PMS de 2014-2017, que contraditóriamente demonstraram, preocupação dos gestores locais em possibilitar uma aproximação com a compreensão das vulnerabilidades sociais presentes no município, apesar de se utilizarem de ações e indicadores com limitações para responderem efetivamente às necessidades em saúde, em especial, dos grupos sociais vulneráveis.

Portanto, é pertinente atuar fortemente na qualificação dos profissionais, visando fomentar a mudança da compreensão dos fenômenos que envolvem o processo de adoecer e morrer das pessoas, o qual é socialmente determinado.

Frente a isso, recomenda-se realização de estudos futuros que proponham metodologias e instrumentos para a ruptura de práticas assistenciais e gerenciais pautadas na multicausalidade e na produtividade em saúde, uma vez que esse arcabouço teóricoprático é limitado para responder às heterogêneas necessidades em saúde, em especial, de grupos sociais vulneráveis.

\section{Referências}

Albuquerque, M.S.V., Costa, A.M., Lima, L.P., \& Melo-Filho, D.A. (2011). Equidade e inclusão de grupos sociais na política de saúde: o caso do Recife, Brasil. Revista Brasileira de Saúde Materno Infantil, 11(1), 73-82. http://doi.org/10.1590/S1519-38292011000100008

Bardin, L. (2015). Análise de conteúdo (1nd ed.). Edições 70.

Brasil. (2016). Conselho Nacional de Saúde. Resolução n. 510, de 7 de abril de 2016. https://bvsms.saude.gov.br/bvs/saudelegis/cns/2016/res0510_07_04_2016.html

Brasil. (2009). Conselho Nacional de Secretarias Municipais de Saúde. Reflexões aos novos gestores municipais de saúde (1st ed.). Conasems. http://bvsms.saude.gov.br/bvs/publicacoes/reflexoes_novos_gestores_municipais.pdf

Breilh, J. (2019). Critical Epidemiology in Latin America: Roots, Philosophical and Methodological Ruptures. In: Vallverdú J., Puyol A., \& Estany A. (Eds.), Philosophical and Methodological Debates in Public Health (pp. 21-45). Springer.

https://www.springer.com/gp/book/9783030286255

Bueno, R.E., Moysés, S.T., Bueno, P.A.R., \& Moysés, S.J. (2013). Governança, sustentabilidade e equidade no plano de saúde de São José dos Pinhais, Brasil. Revista Panamericanam Salud Publica, 34(6), 416-421. https://www.scielosp.org/article/rpsp/2013.v34n6/416-421/

Duncan, P., Bertolozzi, M.R., Cowley, S., Egry, E.Y., Chiesa, A.M., \& Siqueira França, F.O. (2015). 'Health for All' in England and Brazil? International Journal Health Services, 45, 545-563. http://doi.org/10.1177/0020731415584558

Egry, E.Y. (1996). Saúde coletiva: construindo um novo método em enfermagem (1st ed.). Ícone.

Egry, E.Y. (2018). A glance at the Good Practices of Nursing in Primary Care. Revista Brasileira de Enfermagem, 71(3), 930-931. http://doi.org/10.1590/0034-7167.2018710301 
Gatto-Júnior, J.R., Ventura, C.A.A., \& Bueno, S.M.V. (2015). A formação do enfermeiro, o sistema único de saúde e a saúde como um direito: uma revisão sobre a tipologia de conteúdos de ensino-aprendizagem. Arquivos de Ciências da Saúde, 19(1), 59-72. https://doi.org/10.25110/arqsaude.v19i1.2015.5266

Hino, P., Ciosak, S.I., Fonseca, R.M.G.S., \& Egry, E.Y. (2009). Necessidades em saúde e atenção básica: validação de instrumentos de captação. Revista da Escola de Enfermagem da USP, 43(2), 1156-1167. http://doi.org/10.1590/S0080-62342009000600003

Larsen, M., Rantala, R., Koudenburg, A.O., \& Gulis, G. (2014). Intersectoral action for health: the experience of a Danish municipality. Scandinavia Journal Public Health, 42, 649-657. http://doi.org/10.1177/1403494814544397

Martins, L.R., Egry, E.Y., \& Apostólico, M.R. (2015). Consideraciones acerca de la importancia del estudio de saberes necesarios para el afrontamiento de la violencia infantil en la atención primaria de salud. Revista Eletrônica da Faculdade Invest de Ciências e Tecnologia, 14(2), 137-149. https://doi.org/10.22490/25391887.1463

Minayo, Maria Cecilia de Souza, \& Costa, A. P. (2019). Techniques that use speech, observation and empathy: qualitative research in action (1st ed.). Ludomedia.

Nascimento, A.B. (2015). Análise da oferta e da demanda por serviços de saúde de um território sanitário como contribuição para a atenção e gestão em saúde. Revista de Gestão em Sistemas de Saúde, 4(2), 73-86. http://doi.org/10.5585/rgss.v4i2.162

Nascimento, A.B., \& Egry, E.Y. (2017). Municipal health plans and the potentialities of recognition of health needs: a study on four Brazilian Municipalities. Saúde e Sociedade, 26(4), 861-871 http://doi.org/10.1590/s0104-12902017170046

OPAS. (2011). Organização Pan-Americana da Saúde. A atenção à saúde coordenada pela APS: construindo as redes de atenção no SUS (1st ed.). Ministério da Saúde. https://iris.paho.org/handle/10665.2/18457

Santos, A.M., \& Giovanella, L. (2014). Governança regional: estratégias e disputas para gestão em saúde. Revista de Saúde Pública, 48(4), 622-631. http://doi.org/10.1590/S00348910.2014048005045 